\title{
On the Seasonal Variation of Mean Vertical Velocity and Atmospheric Heat-Sources over the Far East from Spring to Summer
}

\author{
by \\ T. Murakami \\ Meteorological Research Institute \\ (Received December 18, 1956)
}

\begin{abstract}
In June, there is a marked zone of downward motion over the southern part of the Sea of Okhotsk, the maximum value of which reaches $10\left(\mathrm{mb} \cdot 12 \mathrm{hr}^{-1}\right)$ at $850-\mathrm{mb}$. It is also apparent that a distinct belt of upward motion extends from the south of Japan eastwards along the latitude circle.

Of considerable significance in the patterns of atmospheric heatsources in June is the existence of a broad zone of cooling over the southern part of the Sea of Okhotsk. The maximum value of this cooling at $850-\mathrm{mb}$ is about $-2 \times 10^{-1}\left(\mathrm{cal} \mathrm{gr}^{-1} 12 \mathrm{hr}^{-1}\right)$.

Such distributions of mean vertical motions and heat-sources show good correspondence with common experience concerning Baiu, which is the rainy season in Japan.
\end{abstract}

\section{Introduction}

It is our main purpose in this paper to obtain some quantitative information about the mean amount of heat added to the air in June over the Far East. In June, the rainy season which is commonly called Baiu visits Japan. OkadA [1] attributed empirically the locality and cause of Baiu to cooling from the cold sea of Okhotsk. Referring to JACOB's work [2], however, which shows the quantity of heat exchanged between the sea and the atmosphere during the four seasons, we cannot detect a distinct cooling from the sea surface near the sea of Okhotsk for the early summer. It becomes, therefore, important to study the quantity of heating in the lower atmosphere in June.

WEXLER [3] determined the normal regions of heating and cooling in the atmosphere from mean circulation data by using the thermodynamic equation. As is well known, there are two unobservable quantities in the thermodynamic equation, i. e. the supply of heat and the vertical velocity. Owing to this circumstance, WEXLER computed only one term expressing horizontal advection of temperature. Therefore, WEXLER's work has suffered from the inability to separate adiabatic warming and cooling due to vertical motion from the true heat sources. Moreover, the strict thermodynamic equation applicable to the mean circulation patterns generally involves the terms expressing the heat transfer due to 
turbulence in horizontal and vertical directions, which are also omitted in WexLeR's computation. It may therefore be said that the quantities of heating and cooling computed by WexLER are composed of three parts-that is, the true heat sources, the adiabatic heating and cooling due to mean vertical velocity, and the apparent heat due to turbulent tranfer.

In order to obtain the mean vertical velocity, we use the so-called mean vorticity equation together with the mean thermodynamic equation. Eliminating the mean height tendency terms between the above two equations, we get the differential equation for the mean vertical velocity. It is a matter of course that in this differential equation the term subject to heat, which is another unobservable quantity, is also included. However, it may be possible to obtain the mean vertical velocity as exactly as possible by taking the differential equation for mean vertical velocity with the mean thermodynamic equation. In doing so, we can easily compute the partial vertical velocity originating from true non-adiabatic heat and also apparent heat.

As for the apparent heat due to turbulent transfer, we have at the present no practical way for estimating this value. Of course this value can be estimated from an empirical and statistical point of view. However, such statistial estimation will make it necessary to carry out enormous computations, which is probably impossible without the aid of the high-speed computor. As this machine is not yet accessible to us, we are unable to separate the apparent heat due to turbulent transfer from the true non-adiabatic heat.

Aubert and Winston [4] studied the relative magnitudes of the apparent heat, and the horizontal and vertical advection of mean temperature due to mean wind speed. The results show that the term expressing the heat transfer due to horizontal turbulence $\left(\overline{\boldsymbol{V}^{\prime} \cdot \bar{\nabla} T^{\prime}}\right)$ is about half that of the horizontal advection of mean temperature $(\overline{\boldsymbol{V}} \cdot \nabla T)$, and the turbulent term $\overline{\left(w^{\prime}\left(\frac{\partial \theta}{\partial \bar{z}}\right)^{\prime}\right)}$ is somewhat small as compared with $\left(\bar{\omega} \frac{\partial \bar{\theta}}{\partial z}\right)$. On account of these results, AubERT and Winston omitted the apparent heat due to turbulence in computing heat sources over the northern hemisphere. However, it was stressed by AuBERT and Winston that these results concerning apparent heat remain quite inconclusive, because of the small sample over a limitted region and also because of an unreliable technique used for computing vertical motion.

\section{The differential equations for the mean vertical velocity and heat}

The vorticity equation for constant pressure surfaces may be expressed in the following form:

$$
\frac{\partial}{\partial t} \nabla^{2} z=J(\eta, z)+\frac{f^{2}}{g} \frac{\partial \omega}{\partial p}+f \frac{\partial}{\partial p}\left(\boldsymbol{k} \cdot \nabla \times \boldsymbol{F}_{M}\right)+K .
$$

Here $z$ is the height of constant pressure surface, $\eta$ the absolute vorticity, $\nabla^{2}$ the horizontal Laplacian operator, $J(\eta, z) \equiv \frac{\partial \eta}{\partial x} \frac{\partial z}{\partial y}-\frac{\partial \eta}{\partial y} \frac{\partial z}{\partial x}, f$ the Coriolis parameter, $g$ the acceleration of gravity, $\omega$ the individual rate of change of pressure $p, k$ the vertical unit vector, and $\boldsymbol{F}_{M}$ the vertical transport of momentum due to turbulence. 
And, $K$ represents the sum of the whole residual terms, such as the vertical advection of vorticity, the transformation of horizontal vorticity into vertical vorticity and so on.

The thermodynamic equation is given by

$$
\begin{aligned}
\frac{\partial}{\partial t}\left(-\frac{\partial z}{\partial p}\right) & =\frac{g}{f} J\left(-\frac{\partial z}{\partial p}, z\right)+\kappa(\omega)+\frac{R}{c_{p} g} \frac{1}{p} q, \\
\kappa & \equiv \frac{\partial z}{\partial p} \frac{\partial \ln \theta}{\partial p},
\end{aligned}
$$

in which $q$ is the supply of heat per unit time and mass, $c_{p}$ the specific heat of dry air, and $R$ the gas constant for dry air.

In order to apply the equations (1) and (2) to normal circulation patterns, it is necessary to take the operation of time averaging on each term in these equations. The normal circulation patterns are defined as mean flow patterns for a very large number of years for one particular month. The resulting mean values may be represented symbolically by placing a bar over each term. Now let each of the quantities-for example the height of isobaric level--be represented by its mean value $\bar{z}$ plus a departure from this mean $z^{\prime}$ :

$$
z=\bar{z}+z^{\prime} .
$$

This expression is introduced into the averaged expression for (1) and (2), and use is made of the fact that in the case of normal circulation patterns the mean value varies much more slowly than the departure from the mean. Then,

$$
\begin{aligned}
& J \overline{(\bar{\eta}, \bar{z})} \fallingdotseq J(\bar{\eta}, \bar{z}), \\
& J \overline{\left(\bar{\eta}, z^{\prime}\right)} \fallingdotseq J\left(\overline{\eta^{\prime}}, \bar{z}\right) \fallingdotseq 0 .
\end{aligned}
$$

Finally, the following equations are obtained:

$$
\begin{aligned}
& \frac{\partial}{\partial t} \Gamma^{2} \bar{z}=J(\bar{\eta}, \bar{z})+\frac{f^{2}}{g} \frac{\partial \bar{\omega}}{\partial p}+f \frac{\partial}{\partial p}\left(\boldsymbol{k} \cdot \nabla \times \overline{\boldsymbol{F}}_{M}\right)+J\left(\overline{\left.\eta^{\prime}, z^{\prime}\right)}+\bar{K},\right. \\
& \frac{\partial}{\partial t}\left(-\frac{\partial \bar{z}}{\partial p}\right)=\frac{g}{f} J\left(-\frac{\partial \bar{z}}{\partial p}, \bar{z}\right)+\bar{\kappa} \bar{\omega}+\frac{R}{c_{p} g} \frac{1}{p} \bar{Q} .
\end{aligned}
$$

Here $\bar{Q}$ is defined by the following expression:

$$
\bar{Q}=\bar{q}+\frac{c_{p} g p}{R}\left[\frac{g}{f} J\left(-\overline{\frac{\partial z^{\prime}}{\partial p}, z^{\prime}}\right)+\overline{\kappa^{\prime} \omega^{\prime}}\right] .
$$

The second term on the right hand side of equation (5) has relation to the transport of heat due to horizontal eddies, and the third term, to the transport of heat due to vertical eddies. Interpreting in a broad sense, it may be possible to regard the second and third terms as representing the heat sources for the normal patterns $\bar{z}$.

Well, the quantity $\frac{\partial V^{2} \bar{z}}{\partial t}$ is usually quite small as compared with each term on the right hand side of the equation (3). For this reason, it is not adequate to use (3) as the prognostic equation. Moreover, we cannot unconditionally 
neglect $\bar{K}$ in (3) for the normal flow patterns, though in the case of short-range numerical prediction the term $K$ in (1) is usually small enough and is neglected.

Eliminating $\partial \vec{z} / \partial t$ between eqs. (3) and (4), we obtain the following equation for mean vertical velocity $\vec{\omega}$ :

$$
\begin{aligned}
\nabla^{2} \bar{\omega}+ & \frac{f^{2}}{g \bar{\kappa}} \frac{\partial^{2} \bar{\omega}}{\partial p^{2}}+\bar{\omega} \nabla^{2} \ln \bar{\kappa}+2(\dot{\nabla} \bar{\omega} \cdot \nabla \ln \bar{\kappa}) \\
= & -(\bar{\kappa})^{-1}\left[\frac{\partial}{\partial p} J(\bar{\eta}, \bar{z})+\frac{g}{f} \nabla^{2} J\left(-\frac{\partial \bar{z}}{\partial p}, \bar{z}\right)+\frac{\partial}{\partial p} J \overline{\left(\eta^{\prime}, z^{\prime}\right)}\right] \\
& -(\bar{\kappa})^{-1}\left[f \frac{\partial^{2}}{\partial p^{2}}\left(\boldsymbol{k} \cdot \nabla \times \overline{\boldsymbol{F}}_{M}\right)+\frac{\partial \bar{K}}{\partial p}\right]-(\bar{\kappa})-1\left[\frac{R}{c_{p} g p} \nabla^{2} \bar{Q}\right] .
\end{aligned}
$$

The boundary condition to be considered will be taken as

$$
\begin{aligned}
& (\bar{\omega})_{p=0}=0, \\
& (\bar{\omega})_{p=p 0}=-\bar{\rho}_{s} g \overline{\boldsymbol{V}}_{s} \cdot \nabla H,
\end{aligned}
$$

where $p_{0}$ is the surface pressure, the subscript $s$ refers to the quantities at the surface, and $H$ the height of the mountain.

If the data for a large number of years, and also the high-speed computer, were available, the values of all terms; except for $\bar{q}$ which is included in $\bar{Q}$, on the right hand side of (6) would be statistically obtained. MinT $z$ [5] calculated the monthly mean values of $J \overline{\left(\eta^{\prime}, z^{\prime}\right)}$ on January and February 1949 over the northern hemisphere at selected isobaric levels. Up to the present, however, the statistical values for $\bar{K}, J\left(\overline{-\frac{\partial z^{\prime}}{\partial p}, z^{\prime}}\right)$, and $\overline{\kappa^{\prime} \omega^{\prime}}$ have not been computed. Moreover, for the present the statistical values concerning water vapour are not available either, so we shall in this paper apparently exclude the heat due to condensation, transport of vapour due to turbulence, and so on.

For the following arguments we shall therefore introduce some approximation upon $J\left(\overline{\left.\eta^{\prime}, z^{\prime}\right)}\right.$ and $K$. Taking a procedure which is similar to that employed in developing the well-known Reynolds stress, we can theoretically approximate $J\left(\overline{\left.\eta^{\prime}, z^{\prime}\right)}\right.$ by the quantities in the mean patterns $\bar{z}$. In order to diminish the error caused by the approximation of $J \overline{\left(\eta^{\prime}, z^{\prime}\right)}$, however, it is more reasonable to connect $J\left(\overline{\eta^{\prime}, z^{\prime}}\right)$ with $J(\bar{\eta}, \bar{z})$ statistically. We shall do this in the simplest way by assuming

$$
\text { (8) } J\left(\overline{\eta^{\prime}, z^{\prime}}\right)=\alpha J(\bar{\eta}, \bar{z})+\text { const. }
$$

Fig. 1 shows the statistical relation between $\nabla^{-2} J\left(\overline{\eta^{\prime}, z^{\prime}}\right)$ and $\nabla^{-2} J(\bar{\eta}, \bar{z})$. The data were obtained from the maps, computed by CLAPP [6] and showing monthly mean patterns of $\nabla^{-2} J\left(\overline{\left.\eta^{\prime}, z^{\prime}\right)}\right.$ and $\nabla^{-2} J(\bar{\eta}, \bar{z})$ in February 1950.

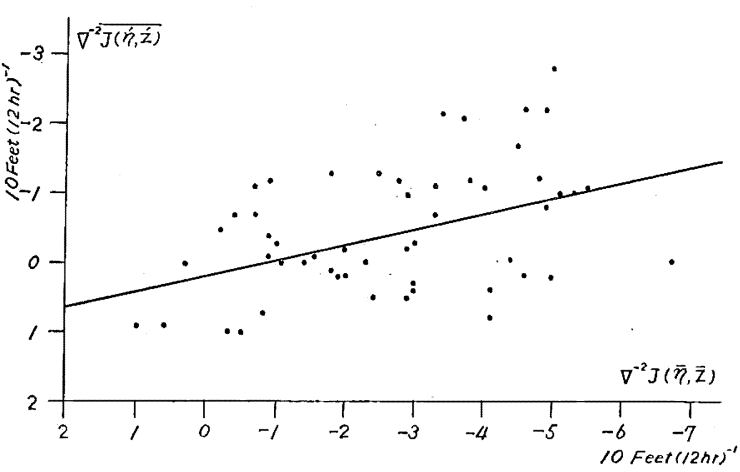

Fig. 1. The statistical relation between monthly mean values of $\nabla^{-2} J\left(\overline{\eta^{\prime}}, z^{\prime}\right)$ and $\nabla^{-2} J(\bar{\eta}, \bar{z})$ in February 1950. 
However, the data over the Pacific were excluded in Fig. 1, because the date are not reliable. Roughly speaking, it is apparent in Fig. 1 that $J\left(\overline{\left.\eta^{\prime}, z^{\prime}\right)}\right.$ linearly correlates with $J(\bar{\eta}, \bar{z})$, and the coefficient of the regression equation is obtained to be

$$
\alpha=0.22 \text {. }
$$

Next, we shall for simplicity assume that the derivative of $\bar{K}$ with $p$ is zero, though $\bar{K}$ may be comparatively large in the vorticity equation (3). That is

$$
\frac{\partial \bar{K}}{\partial p}=0
$$

$K$ in (1) contains the terms such as $\omega \frac{\partial \zeta}{\partial p}$, and $\zeta \frac{\partial \omega}{\partial p}$. It is a matter of common experience that the distribution of $\omega$ with $p$ is nearly parabolic, and $\zeta$ is distributed almost linearly with $p$ in the lower atmosphere. So the distribution of $\omega \frac{\partial \zeta}{\partial p}$, as well as $\zeta \frac{\partial \omega}{\partial p}$, is expressed by some quadratic equation for $p$. Therefore, $\frac{\partial \bar{K}}{\partial p}$, is not generally zero in the lower and upper atmosphere. We have, however, an impression that the error for $\bar{\omega}$ caused by the assumption (9) is probably small. It would be desirable to investigate statistically the nature of $\bar{K}$ in detail. This is being planned.

As the equation (6) is the linear differential equation with respect to $\bar{\omega}$, we can separate $\bar{\omega}$ into three parts as follows:

$$
\bar{\omega}=\bar{\omega}_{1}+\bar{\omega}_{2}+\bar{\omega}_{3} .
$$

After introducing the assumptions (8) and $(9), \bar{\omega}_{i}(i=1,2,3)$ are obtained from the following differential equations :

$$
\begin{gathered}
L\left(\bar{\omega}_{1}\right)=-(\bar{\kappa})^{-1}\left[1.22 \frac{\partial}{\partial p} J(\bar{\eta}, \bar{z})+\frac{g}{f} \nabla^{2} J\left(-\frac{\partial \bar{z}}{\partial p}, \bar{z}\right)\right], \\
\left(\bar{\omega}_{1}\right)_{p=0}=0, \quad\left(\bar{\omega}_{1}\right)_{p=p_{0}}=0, \\
L\left(\bar{\omega}_{2}\right)=-(\bar{\kappa})^{-1} f-\frac{\partial^{2}}{\partial p^{2}}\left(\boldsymbol{k} \cdot \nabla \times \overline{\boldsymbol{F}}_{M}\right), \\
\left(\bar{\omega}_{2}\right)_{p=0}=0, \quad\left(\bar{\omega}_{2}\right)_{p=p_{0}}=-\bar{\rho}_{s} g \overline{\boldsymbol{V}}_{s} \cdot \nabla H, \\
L\left(\bar{\omega}_{3}\right)=-(\bar{\kappa})^{-1} \frac{R}{c_{p} g p} \nabla^{2} \widetilde{Q}, \\
\left(\bar{\omega}_{3}\right)_{p=0}=0, \quad\left(\bar{\omega}_{3}\right)_{p=p_{0}}=0,
\end{gathered}
$$

where $L\left(\bar{\omega}_{i}\right)$ is a linear differential expression of the second order, and is written as

$$
L\left(\bar{\omega}_{i}\right) \equiv \nabla^{2} \bar{\omega}_{i}+\frac{f^{2}}{g \bar{\kappa}}-\frac{\partial^{2} \bar{\omega}_{i}}{\partial p^{2}}+\bar{\omega}_{i} \nabla^{2} \ln \bar{\kappa}+2\left(\nabla \bar{\omega}_{i} \cdot \nabla \ln \bar{\kappa}\right) .
$$

It is here worthwhile to notice that the third and fourth terms on the $r, h, s$ of (14) are sufficiently small in the case of mean flow patterns, and can be neglected with good approximation. Especially the fourth term is quite small 
enough. Strictly speaking, the vertical velocity becomes small in consequence of the effect of the third term in the region where the atmosphere is extremely stable, because $\bar{\kappa}$ is large and then $\nabla^{2} \bar{\kappa}<0$. In the region where the atmosphere is less stable, the reverse is true. The finite difference equation for $\nabla^{2} \ln \bar{\kappa}$ becomes

$$
\nabla^{2} \ln \bar{\kappa}=\frac{1}{d^{2}} \nabla^{2} \ln \bar{\kappa}=\frac{4}{d^{2}}\{[\ln \bar{\kappa}]-\ln \bar{\kappa}\}
$$

in which $d$ is the mesh-size, and [ $\ln \bar{\kappa}]$ denotes the average of $\ln \bar{\kappa}$ in the four neighbouring mesh-points. The numerical values for $\nabla^{2} \ln \bar{\kappa}$ are calculated at various points for January and August. The absolute value for $\nabla^{2} \ln \bar{\kappa}$ does not exceed 0.3. Therefore, the error in computing $\bar{\omega}_{i}$ caused by neglecting the third and fourth terms is roughly less than $1 / 20$ of the value of $\bar{\omega}_{i}$.

It is apparent from eqs. (11), (12) and (13) that $\bar{\omega}_{1}$ represents the mean vertical velocity due to the baroclinicity in the mean field $\bar{z}$, $\bar{\omega}_{2}$, due to the surface friction and the topography, and $\bar{\omega}_{3}$, due to the supply of heat in a broad sense. In such a manner, the method mentioned above makes it possible to see how the various effects contribute to the mean vertical velocity.

It is easy to obtain $\bar{\omega}_{1}$ and $\bar{\omega}_{2}$ from the patterns of each mean isobaric level $\bar{z}$. After obtaining $\bar{\omega}_{1}$ and $\bar{\omega}_{2}, \bar{\omega}_{3}$ can be computed in the following way. Equation (4) may be rewritten in the form :

$$
\frac{\partial}{\partial t}\left(-\frac{\partial \bar{z}}{\partial p}\right)+\frac{g}{f} J\left(\frac{\partial \bar{z}}{\partial p}, \bar{z}\right)-\bar{\kappa}\left(\bar{\omega}_{1}+\bar{\omega}_{2}\right)=\bar{\kappa} \bar{\omega}_{3}+\frac{R}{c_{p} g p} \bar{Q} .
$$

The quantity $\frac{\partial}{\partial t}\left(-\frac{\partial \bar{z}}{\partial p}\right)$ is usually quite small compared to the other terms, and so it will be omitted here for convenience' sake. If the solution of eqs. (11) and (12) are introduced into the $l . h$. $s$ of (4), the sum of each term on the $l . h . s$. becomes a known function of $x, y$ and $p$, which is hereafter expressed as $\vec{\gamma}(x, y, p)$. Therefore, one finds a relation between $\bar{\omega}_{3}$ and $\bar{Q}$ as follows :

$$
\bar{\omega}_{3}=(\bar{n})^{-1} \bar{\gamma}-\frac{R(\bar{n})^{-1}}{c_{p} g p} \bar{Q} .
$$

The differential equation for $\bar{\omega}_{3}$ is now obtained by an elimination $\bar{Q}$ between (13) and (14), giving

$$
\begin{gathered}
\frac{\partial \bar{\omega}_{3}}{\partial p^{2}}=-\frac{g}{f^{2}} \nabla^{2} \bar{\gamma}, \\
\left(\bar{\omega}_{3}\right)_{p=0}=0, \quad\left(\bar{\omega}_{3}\right)_{p=0}=0 .
\end{gathered}
$$

The solution of (15) is easily obtained, and is written as

$$
\bar{\omega}_{3}=\frac{g}{f^{2}}\left(1-\frac{p}{p_{0}}\right) \int_{0}^{P} \int_{0}^{P} \nabla^{2} \bar{\gamma} d p^{\prime} d p-\frac{g}{f^{2}} \int_{p}^{P} 0 \int_{p}^{P}{ }^{P} \nabla^{2} \bar{\gamma} d p^{\prime} d p
$$

Finally $\bar{Q}$ at any isobaric level is computed to be

$$
\bar{Q}=\frac{c_{p} g p}{R}\left[\bar{\gamma}-\bar{\kappa} \bar{\omega}_{3}\right] .
$$


As is easily seen in (14), the effect coming from the apparent heat due to turbulent transfer is also included in $\bar{\omega}_{3}$.

\section{Numerical integration}

In this section we shall discuss the practical procedure for getting $\bar{\omega}$ and $\bar{Q}$ at any isobaric level. The data of the mean isobaric heights in the upper atmosphere (higher than $500-\mathrm{mb}$ level) are not available at present, so we can not, strictly, solve the differential equations mentioned in the previous section. It becomes now necessary to introduce some approximate method.

First, we shall describe the method for computing $\bar{\omega}_{1}$. In the Normal Weather Charts published by the U.S. Weather Bureau, the monthly mean normal patterns

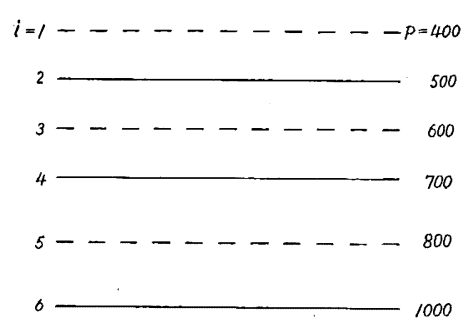

Fig. 2. Schematical indication of locations of each isobaric level. for isobaric heights at $500-\mathrm{mb}, 700-\mathrm{mb}$, and $1000-\mathrm{mb}$ are drawn. Since the values of the source terms on the $r . h$. $s$ of (11) at the isobaric levels, which are higher than $500-\mathrm{mb}$ level, are not given, it is impossible to solve (11) subject to the strict upper boundary condition, say $\left(\bar{\omega}_{1}\right)_{p=0}=0$. So we put the vertical velocity at $600 \mathrm{mb}$, that is $\left(\bar{\omega}_{1}\right)_{600}$, as an approximate value. It is our purpose to compute $(\bar{\omega})_{850}$ by making use of $\left(\bar{\omega}_{1}\right)_{600}$ as the upper boundary condition. As can easily be seen in (11), the general solution of a homogeneous equation decreases exponentially with $p$ in its value. It is, therefore, expected that the approximation for $\left(\bar{\omega}_{1}\right)_{600}$ causes the small error for the estimation of $\left(\bar{\omega}_{1}\right)_{850}$. One method to obtain the approximate value of $\left(\bar{\omega}_{1}\right)_{600}$ is to use the prabolic assumption, which is given by

$$
\left(\bar{\omega}_{1}\right)_{600}=\frac{4 p}{p_{0}}\left(1-\frac{p}{p_{0}}\right) \omega^{*}
$$

in which $p=600 \mathrm{mb}$. Substituting this expression into (11), we get the differential equation for $\omega^{*}$ as follows :

$$
\begin{aligned}
\nabla^{2} \omega^{*} & -\frac{f^{2}}{g \bar{\kappa}} \frac{2}{p_{3}\left(p_{0}-p_{3}\right)} \omega^{*} \\
& =-(\bar{\kappa})^{-1} \frac{p_{0}{ }^{2}}{4 p_{3}\left(p_{0}-p_{3}\right)}\left[1.22 \frac{\partial}{\partial p} J(\bar{\eta}, \bar{z})+\frac{g}{f} \nabla^{2} J\left(-\frac{\partial \bar{z}}{\partial p}, \bar{z}\right)\right],
\end{aligned}
$$

where $p_{3}$ equals $600 \mathrm{mb}$. The source term on the r.h.s may be evaluated by using the data at two isobaric levels of $500 \mathrm{mb}$ and $700 \mathrm{mb}$.

Introducing a finite difference for the vertical derivative with $p$ on the $l$. h. $s$ of (11) and taking the lower boundary condition into consideration, $\left(\bar{\omega}_{1}\right)_{850}$ is now given as follows :

$$
\begin{aligned}
\nabla^{2}\left(\bar{\omega}_{1}\right)_{850} & -\frac{f^{2}}{g \bar{\kappa}} \frac{2}{\left(p_{6}-p_{5}\right)\left(p_{5}-p_{3}\right)}\left(\bar{\omega}_{1}\right)_{850} \\
& =-(\bar{\kappa})-1\left[1.22 \frac{\partial}{\partial p} J(\bar{\eta}, \bar{z})+\frac{g}{f} \nabla^{2} J\left(-\frac{\partial \bar{z}}{\partial p}, \bar{z}\right)\right]-\frac{f^{2}}{g \bar{\kappa}} \frac{2\left(\bar{\omega}_{1}\right)_{600}}{\left(p_{6}-p_{3}\right)\left(p_{5}-p_{3}\right)},
\end{aligned}
$$


in which $p_{i}$ indicates the respective values of pressure corresponding to each isobaric level. The first term on the $r . h . s$ is given by using the data at two isobaric levels of $700 \mathrm{mb}$ and $1000 \mathrm{mb}$. Solving Helmholtz's equation (20), $\left(\bar{\omega}_{1}\right)_{850}$ can easily be obtained.

Next, the method to obtain $\left(\bar{\omega}_{2}\right)$ will be outlined. The vertical flux of momentum due to turbulence $\boldsymbol{F}_{M}$ may simply be written in the following form:

$$
\overline{\boldsymbol{F}}_{M}=\bar{\rho}^{2} g k_{M^{-}} \partial \overline{\overline{\boldsymbol{V}}}
$$

here $\rho$ is the density, $k_{M}$ the Austausch coefficient, $\boldsymbol{V}$ the wind vector. It is reasonable to assume that the frictional drag is negligible above the $850-\mathrm{mb}$ isobaric level. Assuming that, and introducing the finite difference for the vertical derivative with $p$ in the r.h.s of (12), we get the following relation:

$$
\left(\frac{\partial^{2}}{\partial p^{2}}\left[\boldsymbol{k} \cdot \nabla \times \overline{\boldsymbol{F}}_{M}\right]\right)_{850}=\frac{2}{\left(p_{6}-p_{3}\right)\left(p_{6}-p_{5}\right)}\left[\boldsymbol{k} \cdot \nabla \times \overline{\boldsymbol{F}}_{M}\right]_{1000} .
$$

Now, we may approximately rewrite the term on the $r . h . s$ by using (21) in the form

$$
\left[\boldsymbol{k} \cdot \nabla \times \overline{\boldsymbol{F}}_{M}\right]_{1000}=\left[\bar{\rho}^{2} g k_{M} \frac{\partial \bar{\zeta}}{\partial p}\right]_{1000} \fallingdotseq-\left[\bar{\rho} k_{M} \frac{\partial \bar{\zeta}}{\partial z}\right]_{z=0},
$$

in which $\zeta$ is the relative vorticity.

It is not difficult to obtain the vertical gradient of relative vorticity at the ground. An approximate solution of wind speed in the friction layer has been proposed by Syōno [7], subject to the following boundary condition :

$$
\begin{array}{ll}
z=0 ; & \boldsymbol{V}=m \frac{d \boldsymbol{V}}{d z}, \\
z=\infty ; & \boldsymbol{V}=\text { finite },
\end{array}
$$

where $m$ is the proportional constant. The result of Syōno takes the form

$$
\begin{gathered}
u=u_{0}\left\{1+C e^{-B z} \sin (B z-s)\right\}-v_{0} C e^{-B z} \cos (B z-s), \\
v=v_{0}\left\{1+C e^{-B z} \sin (B z-s)\right\}+u_{0} C e^{-B z} \cos (B z-s), \\
2 B^{2}=f / k_{M}, \quad \tan s=\frac{1+m B}{m B}, \\
C=1 / \sqrt{1+2 m B+2 m^{2} B^{2}},
\end{gathered}
$$

in which $u_{0}$ and $v_{0}$ are the components of the geostrophic speed at the ground in the eastward and the northward directions, respectively.

After calculating the relative vorticity from the above equation, differentiating with respect to $z$ and putting $z=0$, we now obtain the following equation with good approximation :

$$
\begin{aligned}
{\left[\frac{\partial \bar{\zeta}}{\partial z}\right]_{z=0} } & \doteqdot C B(\sin s+\cos s)(\bar{\zeta})_{z=0} \\
& \fallingdotseq C B(\sin s+\cos s)\left(\bar{\zeta}_{1000} .\right.
\end{aligned}
$$


Finally, we obtain the following relation:

$$
\left[k \cdot \nabla \times \boldsymbol{F}_{M}\right]_{1000}=-\bar{\rho}_{6} k_{M} C B(\sin s+\cos s) \bar{\zeta}_{1000},
$$

in which $\rho_{6}$ is the density at $1000 \mathrm{mb}$.

It is more or less complicated to treat the lower boundary condition of (12) strictly, because there are regions where the mountain, such as the Himalaya, is higher than isobaric level of $850 \mathrm{mb}$ or $600 \mathrm{mb}$. The lower boundary condition may be rewritten as

$$
\left(\bar{\omega}_{2}\right)_{p=p_{s}}=\frac{\bar{\rho}_{s} g^{2}}{f} J\left(H, \bar{z}_{s}\right),
$$

in which $p_{s}=p_{s}(x, y)$ is the pressure on the ground surface, and $\bar{z}_{s}$ is the isobaric height at the ground. $\bar{\rho}_{s}$ and $\bar{p}_{s}$ are estimated from the normal state of the atmosphere in accordance with the height of the mountain at the point under consideration. The height of mountain $H(x, y)$ is determined from the mean tropographic charts, which are established by BERKoFsKy and BERTONi [8], and show the smoothed profile of the height of large-scale mountains.

Over the region where the mountain is not higher than the normal height of the $700-\mathrm{mb}$ isobaric surface, it is natural to approximate $\bar{z}_{s}$ as

$$
\bar{z}_{s}=\bar{z}_{1000}+\frac{\delta p}{\Delta p} \bar{h}
$$

Here $\bar{h}=\bar{z}_{700}-\bar{z}_{1000}, \Delta p=1000 \mathrm{mb}-700 \mathrm{mb}, \delta p=1000 \mathrm{mb}-p_{s}$. Making use of (27), (26) becomes

$$
\left(\bar{\omega}_{2}\right)_{p=p_{s}}=\frac{\bar{\rho}_{s} g^{2}}{f} J\left(H, \bar{z}_{1000}+\frac{\delta p}{\Delta p} \bar{h}\right) .
$$

We find $\left(\bar{\omega}_{2}\right)_{p=p_{s}}$ in the region, where the mountain is higher than the $700-\mathrm{mb}$ isobaric level, by the same treatment as mentioned above.

Strictly speaking, the surface of the lower boundary in (12) does not coincide with one isobaric surface, but with the shape of the mountain. On the other hand, the Normal Weather Charts published by the U.S. Weather Bureau are drawn over the entire northern hemisphere, without regard to the height of the mountain. It may, therefore, be convenient to regard $\left(\bar{\omega}_{2}\right)_{p=p_{s}}$ given in (28) as the vertical velocity at $1000 \mathrm{mb}$. Perhaps, this assumption will not bring about any serious errors over the main part of the domain under consideration, because we are mainly concerned with the vertical velocity near Japan which is situated far from the Himalayas.

Under the assumption mentioned above and upon introducing the finite difference for the vertical derivative on the $l . h . s$ of (12), it is easy to show the eq. (12) can be written as

$$
\left\{\begin{array}{l}
\nabla^{2}\left(\bar{\omega}_{2}\right)_{850}-\frac{f^{2}}{g \bar{\kappa}} \frac{2}{\left(p_{6}-p_{5}\right)\left(p_{5}-p_{3}\right)}\left(\bar{\omega}_{2}\right)_{850}+\frac{f^{2}}{g \bar{\kappa}} \frac{2}{\left(p_{6}-p_{3}\right)\left(p_{5}-p_{3}\right)}\left(\bar{\omega}_{2}\right)_{600} \\
=(\bar{\kappa})^{-1} F \frac{4}{\left(p_{6}-p_{3}\right)\left(p_{6}-p_{5}\right)} \nabla^{2} z_{1000}-\frac{2}{\left(p_{6}-p_{3}\right)\left(p_{6}-p_{5}\right)}(\bar{\omega})_{p=p_{s}}
\end{array}\right.
$$




$$
\left(\nabla^{2}\left(\bar{\omega}_{2}\right)_{600}-\frac{f^{2}}{g \bar{\kappa}} \frac{2}{\left(p_{5}-p_{3}\right)\left(p_{3}-p_{1}\right)}\left(\bar{\omega}_{2}\right)_{600}+\frac{f^{2}}{g \bar{\kappa}} \frac{2}{\left(p_{5}-p_{1}\right)\left(p_{5}-p_{3}\right)}\left(\bar{\omega}_{2}\right)_{850}=0,\right.
$$

where $F=\bar{\rho}_{6} g k_{M} C B(\sin s+\cos s)$. The above equations are derived by putting the upper boundary condition as $\left(\bar{\omega}_{2}\right)_{400}=0$ in stead of using $\left(\bar{\omega}_{2}\right)_{p=0}=0$. Because of the nature of the boundary condition described before, the boundary condition $\left(\bar{\omega}_{2}\right)_{400}=0$ will scarcely cause any error in estimating $\left(\bar{\omega}_{2}\right)_{850}$.

Finally we shall describe the simple method for getting $\bar{\omega}_{3}$ and $\bar{Q}$. As was shown in the above, $\bar{\omega}_{1}$ and $\bar{\omega}_{2}$ are only given at two isobaric levels of $600 \mathrm{mb}$ and $850 \mathrm{mb}$. Referring to the definition of $\bar{\gamma}$ proposed in the previous section, the quantities $\bar{\gamma}$ are also given at two confined isobaric levels. It is, therefore, impossible to compute the first term on the $r . h . s$ of eq. (16) strictly, which is the solution of the differential equation for $\bar{\omega}_{3}$. So we put the vertical velocity $\bar{\omega}_{3}$ at $600 \mathrm{mb}$ as an approximate value, corresponding to $\bar{\gamma}$ at $600 \mathrm{mb}$. The approximate value of $\left(\bar{\omega}_{3}\right)_{600}$ is gained in the same manner as that used for $\left(\bar{\omega}_{1}\right)_{600}$, and is given by

$$
\left(\bar{\omega}_{3}\right)_{600}=\frac{g}{f^{2}} \frac{p_{3}\left(p_{6}-p_{3}\right)}{2} \nabla^{2}(\bar{\gamma})_{600}
$$

where $p_{3}=600 \mathrm{mb}$.

Introducing a finite difference for the vertical derivative with $p$ on $l . h$. s. of eqi (15) and taking the lower boundary condition into consideration, $\left(\bar{\omega}_{3}\right)_{850}$ is easily obtained as follows :

$$
\left(\bar{\omega}_{3}\right)_{850}=-\stackrel{g}{f^{2}} \frac{\left(p_{6}-p_{5}\right)\left(p_{5}-p_{3}\right)}{2} \nabla^{2}(\bar{\gamma})_{850}+\frac{p_{6}-p_{5}}{p_{6}-p_{3}}\left(\bar{\omega}_{3}\right)_{600} .
$$

Substituting (30) into (31), (31) may be rewritten as follows :

$$
\left(\bar{\omega}_{3}\right)_{850}=\frac{g\left(p_{6}-p_{5}\right)}{2 f^{2}}\left[\left(p_{5}-p_{3}\right) \nabla^{2}(\bar{\gamma})_{850}+p_{3} \nabla^{2}(\bar{\gamma})_{600}\right]
$$

In the above equation, all coefficients of $\nabla^{2}(\bar{\gamma})_{i}(i=600,850)$ are positive. Thus, the sign of the vertical velocity due to the heat depends directly upon $\nabla^{2}(\bar{\gamma})_{i}$, the sign of which is usually opposite to that of $(\bar{\gamma})_{i}$. As can be seen in $(17),(\tilde{\gamma})_{i}$ is roughly proportional to $\bar{Q}_{i}$ which is the heat in a broad sense. It may, therefore, be possible to suggest that the vertical motion due to heat is upward in the region occupied by warm source and is downward within the cold source region.

The proper values we adopted for various constants that appeared in the above are the following:

$$
\begin{aligned}
& d=600 \mathrm{~km}, \quad k_{M}=10 \mathrm{~m}^{2} \mathrm{sec}^{-1}, \quad c_{p}=0.24 \mathrm{cal}(\mathrm{gr})^{-1}, \\
& C=0.94 \quad B=1.24 \times 10^{-3} \mathrm{~m}^{-1} \text {. }
\end{aligned}
$$

The values of $\bar{\kappa}$ usually varies with $x, y$, and $p$. However, we assume for simplicity that $\bar{k}$ is a function of $p$ alone. Moreover the value of $\bar{\kappa}$ differs from month to month. The values of $\vec{k}$ are evaluated from the monthly mean state at Tateno in Japan, and are shown in Table 1. 
Table 1. Monthly mean values of $\bar{\kappa}$ in $10^{-3} \mathrm{~m}(\mathrm{mb})^{-2}$.

\begin{tabular}{l|r|r|r|r|r|r}
\hline & Jan. & Apr. & May & June & July & Aug. \\
\hline$(\bar{\kappa})_{600}$ & 3.0 & 2.8 & 2.8 & 3.2 & 3.2 & 3.0 \\
$(\bar{\kappa})_{850}$ & 1.6 & 1.3 & 1.3 & 1.5 & 1.4 & 1.4 \\
\hline
\end{tabular}

\section{Discussion of results}

It was shown by JAcoBs [2] that in the cold season vast amounts of sensible heat are supplied to the atmosphere from the sea surface in the western Pacific. And the marked maximum in the supply of heat is encountered just east of the Japanese islands. It is therefore interesting to compare the distributions of the supply of heat proposed by JACOBS with those of heat-sources at lower atmosphere obtained by our method in January.

As a check of the method used here, we first computed the mean vertical velocity and the patterns of heating and cooling in January. In Figs. 3 (a) and (b),

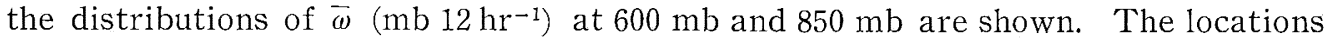
of grids the interval of which is $600 \mathrm{~km}$ are also shown in Fig. 3 (a).

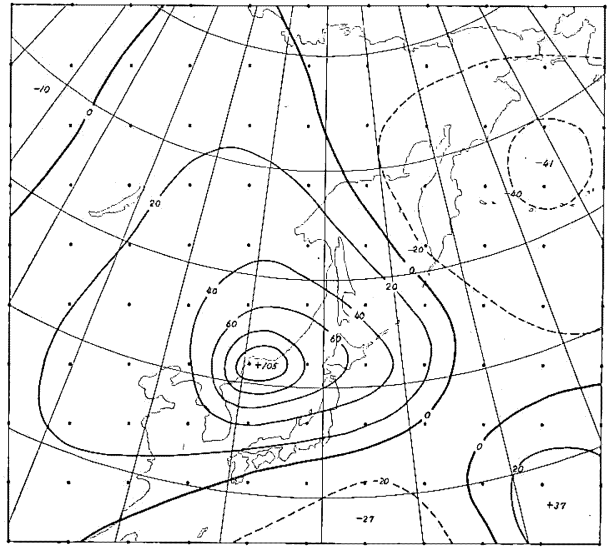

(a) $600 \mathrm{mb}$

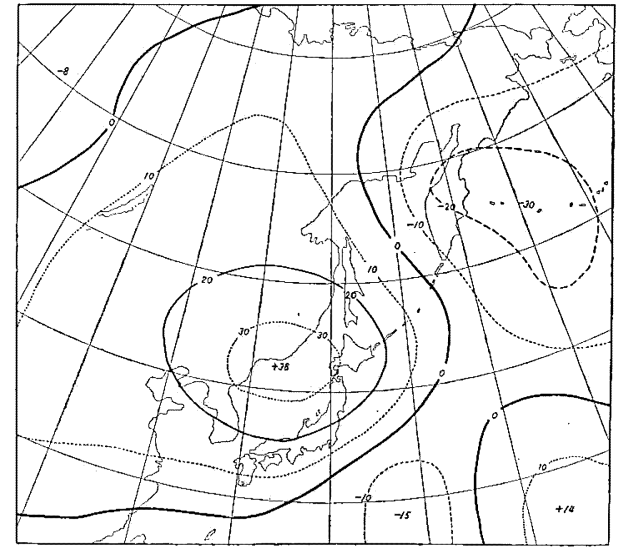

(b) $850 \mathrm{mb}$

Fig. 3. The mean vertical velocity $\left(\mathrm{mb} \cdot 12 \mathrm{hr}^{-1}\right)$ for January. The distributions of upward motion are shown by dashed lines, and those of downward motion, by continuous lines. Isolines are drawn at $20 \mathrm{mb} \cdot 12 \mathrm{hr}^{-1}$ intervals. The locations of finite-difference latice used in computation are also shown. Dotted lines are drawn at $10 \mathrm{mb} \cdot 12 \mathrm{hr}^{-1}$ intervals.

It is immediately apparent that a broad area of relatively large downward motion extends over the northwestern part of the Japan Sea at both $600 \mathrm{mb}$ and

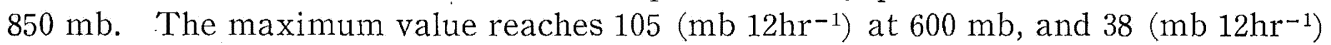
at $850 \mathrm{mb}$ which is about one third of the former. At any rate, it is of interest to point out that in January there is a marked downward motion over the entire Japanese islands. It is worthwhile here to add that in this paper the small-scale roughness of the earth's surface is neglected, and then the forced upward motion 
due to the so-called northwesterly monsoon at the western side of the Japanese islands is excluded in Fig. 3. Over the Aleutian islands and also over the south of the Japanese islands, a pronounced upward motion occurs. The upward motion centering at the middle of the Aleutian islands may be supposed to correspond closely with the well-known Aleutian low.

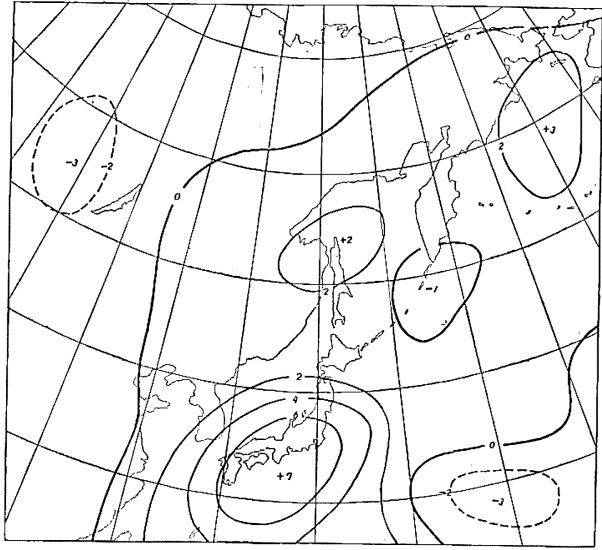

(a) $600 \mathrm{mb}$

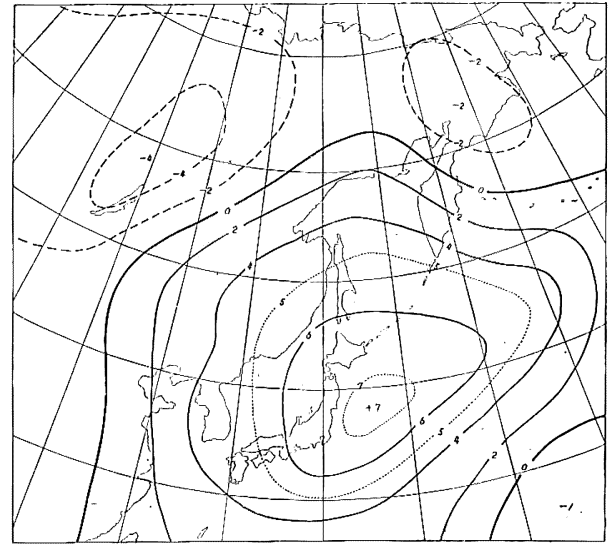

(b) $850 \mathrm{mb}$

Fig. 4. Mean amount of heat $\left(10^{-1} \cdot\right.$ cal $\left.\cdot \mathrm{gr}^{-1} \cdot 12 \mathrm{hr}^{-1}\right)$ for January at $600 \mathrm{mb}$, and $850 \mathrm{mb}$. Dashed lines are drawn for cooling, and continuous lines for heating. Isolines are for $2 \times 10^{-1} \cdot \mathrm{cal} \cdot \mathrm{gr}^{-1} \cdot 12 \mathrm{hr}^{-1}$ intervals. Dotted lines are drawn at $1 \times 10^{-1} \cdot \mathrm{cal} \cdot \mathrm{gr}^{-1} \cdot 12 \mathrm{hr}^{-1}$ intervals.

Figs. 4 (a) and (b) show the supply of heat in a broad sense at $600 \mathrm{mb}$ and $850 \mathrm{mb}$ respectively. It may, however, happen that the patterns of $(\bar{Q})_{600}$ will involve some error, as pointed out in the previous section. Therefore Fig. 4 (a) is here shown only for the purpose of supplement. Glancing at Fig. 4 (b), it is at once apparent that there is a pronounced heating over the western part of the Pacific centering at just east of the Japanese islands, which is much the same position as that in the figure drawn by JAcoBs. Another characteristic feature in Fig. 4 (b) is the fact that cooling predominates over the continent. The center of cooling is located slightly north of the lake of Baikal, which fairly corresponds with the center of the Siberian high.

It is of interest that the distribution of $(\bar{Q})_{850}$ shows good correspondance with common experience concerning the heat supply from the ground surface. Of course, it is easy to take into consideration theoretically the supply of heat from the ground surface in our computation in the same manner as JACoBs did, which depends on assumed heat exchange coefficient and the temperature difference between sea and air. But information on these quantities on the land surface is generally insufficient and moreover the magnitude of the Austausch coefficient for the vertical transport of heat depends largely on the wind speed and the lapse rate adjacent to the earth's surface. Under these circumstances, we put out of account in this paper supply of heat from the earth's surface. 
Since the sensible heat supplied from the sea or land is concentrated near the earth's surface, turbulent vertical transport up to rather high levels of the troposphere may account for much of the sizable values of heating found in Fig. 4 . At any rate, it is natural to suppose that the patterns of $(\bar{Q})_{850}$ shown in Fig. 4 (b) depend largely upon the transport of heat from warmer sea surface or colder land surface due to vertical eddies, which is included in $\bar{Q}$ as shown in eq. (5). At present, however, we cannot discuss this problem quantitatively.

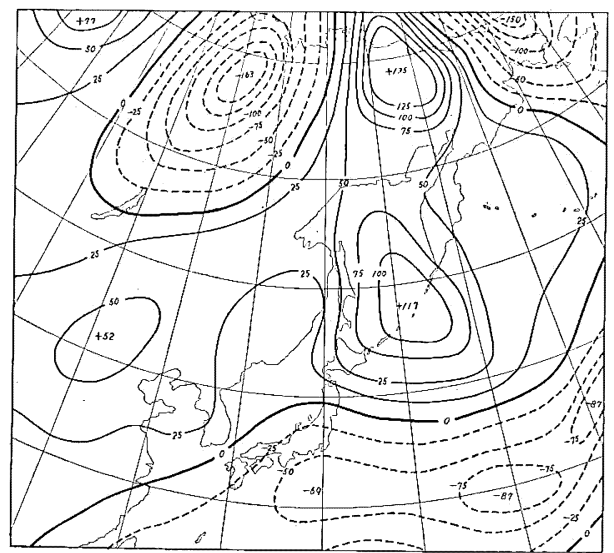

(a) $600 \mathrm{mb}$

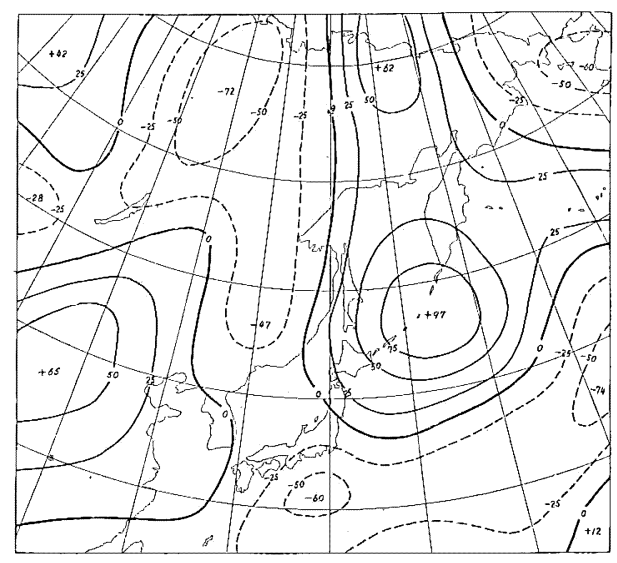

(b) $850 \mathrm{mb}$

Fig. 5. The mean vertical velocity $\left(10^{-1} \cdot \mathrm{mb} \cdot 12 \mathrm{hr}^{-1}\right)$ for June at $600 \mathrm{mb}$, and $850 \mathrm{mb}$. The distribution of upward motion are shown by dashed lines, and those of downward motion by continuous lines. Isolines are drawn at $25 \times 10^{-1}$. $\mathrm{mb} \cdot 12 \mathrm{hr}^{-1}$, intervals.

Figs. 5 (a) and (b) show the distribution of mean vertical velocity in June at $600 \mathrm{mb}$ and $850 \mathrm{mb}$ respectively. One striking feature in these figures is the fact that there is a marked zone of downward motion over the central Kurile islands, and there is a remarkable belt of upward motion stretching from the south of Japan to the east along the latitude circle. By the way, in June the rainy season called Baiu visits Japan, which generally starts in the beginning of June and comes to an end in the middle of July. During the Baiu season, the surface map is characterized by the circumstances that there is a somewhat persistent anticyclone over the sea of Okhotsk which is commonly called the Okhotsk high, and also that there is a stationary front stretching eastwards from just south of Japan, which is called the Baiu front. Thus it may be supposed that the zone of downward motion centering at the central Kurile islands corresponds to the Okhotsk high, and the belt of upward motion mentioned above is closely connected with the Baiu front.

Turning our attention now to the features in higher latitudes in Fig. 5, it is at once apparent that there is a wave train of upward and downward motions alternately. Such distribution of vertical motions is fairly in accordance with the common experience, namely, that there is upward motion to the east of the mean trough in upper westerlies and downward motion to the east of the mean wedge. The maximum value of downward or upward motion at $850 \mathrm{mb}$ is smaller than 
that at $600 \mathrm{mb}$ by about one half. On the other hand, the magnitude of the downward motion centering at the central Kurile islands, as well as that of the upward motion stretching in the south of Japan, is nearly equal at $850 \mathrm{mb}$ and at $600 \mathrm{mb}$. This suggests the different dynamical structure of the upper atmosphere between high and middle latitudes in the Baiu season.

Fig. 6 presents the distribution of mean vertical velocity at $850 \mathrm{mb}$ due to heating defined in the previous section. Broadly speaking, the vertical motion due

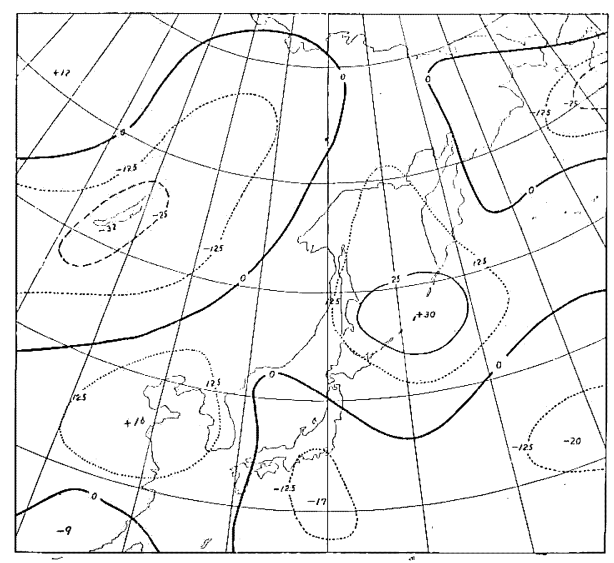

Fig. 6. The mean vertical velocity due to heat for June at $850 \mathrm{mb}$. Dotted lines are drawn at $12.5 \times 10^{-1} \cdot \mathrm{mb}$. $12 \mathrm{hr}^{-1}$ intervals. (Refer to Fig. 5 for further explanation). to heating $\bar{\omega}_{3}$ is upward in the region occupied by the warm source, and is downward within the cold source region, as pointed out before. So it is no wonder that the patterns of $\left(\bar{\omega}_{3}\right)_{850}$ resemble those of $(\bar{Q})_{850}$ drawn in Fig. 7 (b) in their features. Comparison of Fig. 6 with Fig. 5 (b) shows that the vertical velocity due to heat is about one third of the total vertical velocity. It is of interest here to point out that the magnitude of $\bar{\omega}_{3}$ is comparatively large.

Figs. 7 (a) and (b) show the patterns of the heat defined in eq. (5) in June. Of considerable significance in Fig. 7 (b) is the existence of a broad zone of cooling centering over the southern part of the sea of Okhotsk. OKADA [1] empirically attributed the locality and the cause of Baiu to cooling from the cold sea of Okhotsk, which is favourable for the formation of a persistent anticyclone there. According to the Atlas of Climatic

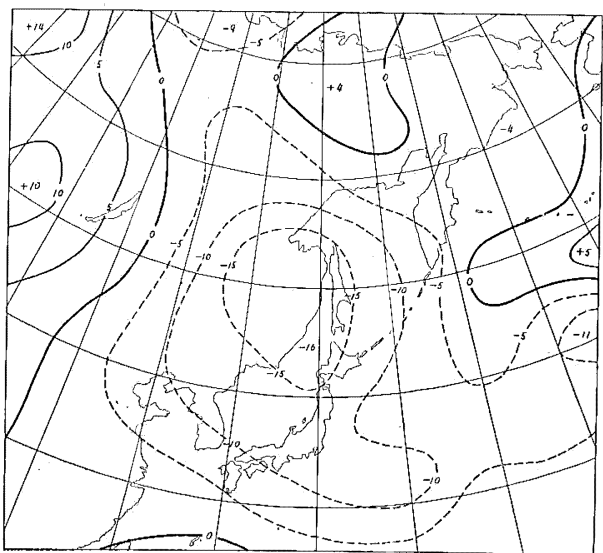

(a) $600 \mathrm{mb}$

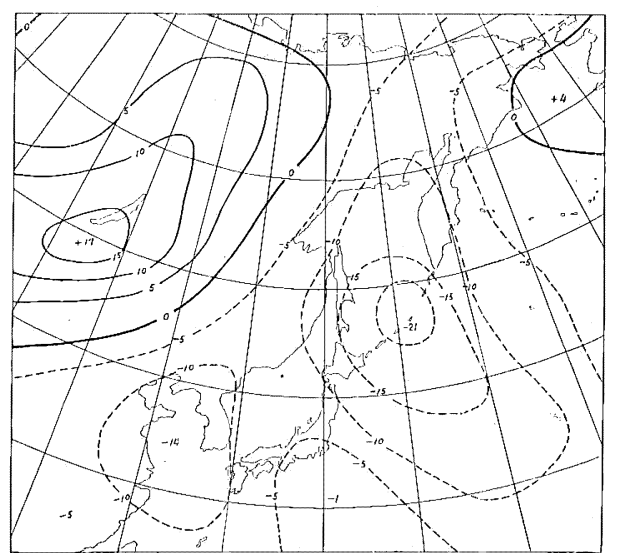

(b). $850 \mathrm{mb}$

Fig. 7. Mean amount of heat for June. Dashed lines are drawn for cooling, continuous lines for heating. Isolines are for $5 \times 10^{-1} \cdot \mathrm{cal} \cdot \mathrm{gr}^{-1} \cdot 12 \mathrm{hr}^{-1}$. 
Charts of the Oceans published by the U. S. Weather Bureau, however, we cannot detect a distinct temperature difference between air and sea in June. The maximum temperature difference reaches only about $2^{\circ} \mathrm{F}$. In January, however, this value exceeds $10^{\circ} \mathrm{F}$.

On account of indistinct appearance of the temperature difference between sea and air, we cannot expect that the zone of cooling occuring over the sea of Okhotsk in Fig. 7 (b) is fully associated with cooling from the sea surface, though in January such association is undoubtedly recognized. Therefore, it is necessary to seek for other mechanisms concerning the supply of heat in June. We shall again discuss this problem later.

Another striking features in Fig. 7 (b) is the fact that there is a remarkable zone of heating over the continents. Perhaps this heating is closely associated with the heating from the warmer land surface. As will be seen later, the magnitude of heating over the continent is the largest in June for the period from April to August. Moreover, the magnitude of cooling over the oceans is also the largest in June. So there is in June the maximum contrast between heating over the continent and cooling over the oceans, which is probably favourable for the formation of an anticyclone over the sea of Okhotsk and the formation of a cyclone over the continent by thermal effect.

Now we shall turn our attention to the seasonal variation of mean vertical velocity in the lower atmosphere from spring to summer. Four figures, Fig. $8 \mathrm{a}$ to $8 \mathrm{~d}$ are drawn in order to get clear information about such seasonal variation. One characteristic feature in April is the existence of a broad zone of downward motion extending from northeastern China to northern Siberia, as is seen in Fig. 8a.

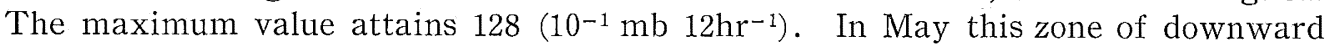
motion separates into two, one situated in northeastern China, which is much the same position as in April, and the other over the northwestern Pacific. As for the latter, the maximum downward motion is found over the central Kurile islands, which is the same position as that in June, though the maximum value is only about half of that in June. The separation of downward motion becomes most remarkable in June, as is seen in Fig. 5 (b). On a glance at Fig. 8c, it is apparent that two separated zones of downward motion remain till July in their respective positions. In August, however, one of these zones shifts from the central Kurile islands to the northeastern periphery of Siberia. The author has an impression that this shift happens at the same time with the disappearance of the so-called Okhotsk high-that is to say, at the time of the end of Baiu.

Next, we shall discuss the change in the belt of upward motion, which is clearly seen in Fig. 8a and is extending from Formosa to the Bering Sea. This belt seems to shift gradually northwards as the season advances. Owing to this northward shift, there comes to exist upward motion over the main part of Japanese islands in June, as is seen in Fig. 5 (b). In July, this belt of upward motion becomes indistinct near Japan. And it may be said that in August this belt almost disappears.

As is seen in Fig. 8b, another belt of upward motion seems to extend in higher latitudes through the Arctic Ocean. Such a belt of upward motion can be recognized during the period from April to July. It was previously pointed out by the author [9] that over the Far East two belts of jet streams appear in April, and disappear 


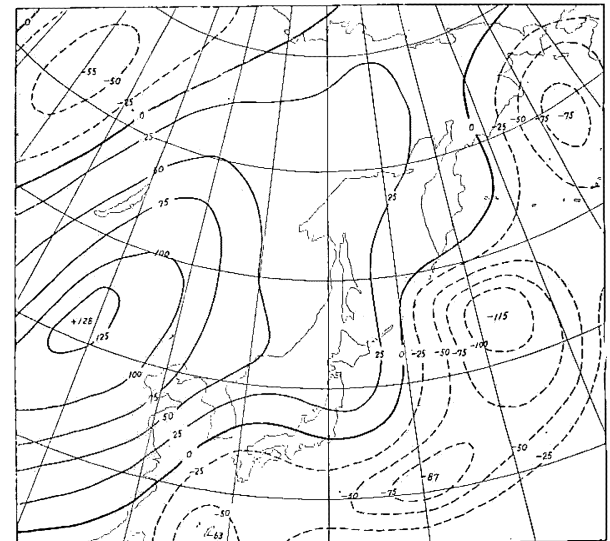

(a) April

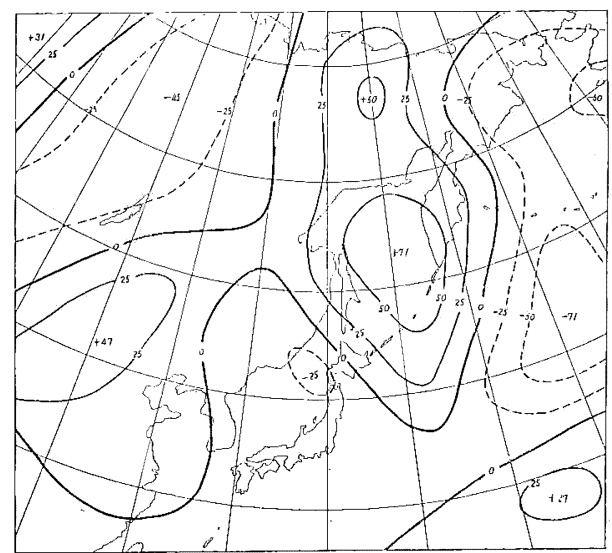

(c) July

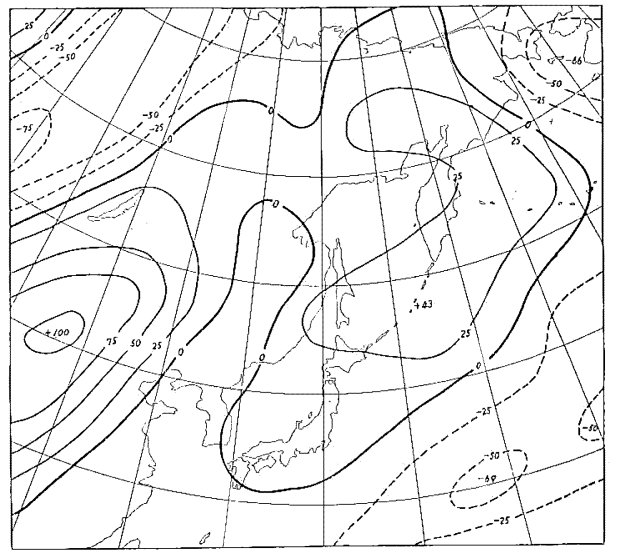

(b) May

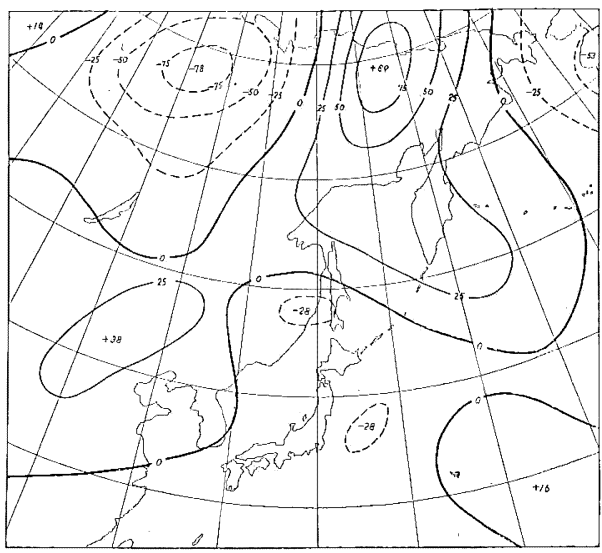

(d) August

Fig. 8. Mean vertical velocity at $850 \mathrm{mb}$. (Refer to Fig. 5 for further explanation).

at the time of the end of Baiu. The locations of the two belts of jet streams are almost the same as those of upward motions montioned above. It is, therefore, reasonable to suppose that the two belts of upward motion are closely connected with the two belts of jet streams. Referring to the discussion heretofore, we must lay stress on the fact that the patterns of mean vertical velocity which are similar to those in Baiu already appear before May. This fact, too, suggests that the cause of Baiu is not merely the cooling from the cold sea of Okhotsk.

The distributions of heating and cooling sources at $850 \mathrm{mb}$ during the period from April to August except for June are shown in Fig. 9. It will be seen in these figures that during this period distinct heating source extends over the continent. The extent, as well as the magnitude, of this heating source is almost the same through the period, though the magnitude becomes slightly large and attains $17 \times 10^{-2}$ (cal $\left.\mathrm{gr}^{-1} 12 \mathrm{hr}^{-1}\right)$ in May and June. And the location of the center of heating source remains near the lake of Baikal from spring to summer. 


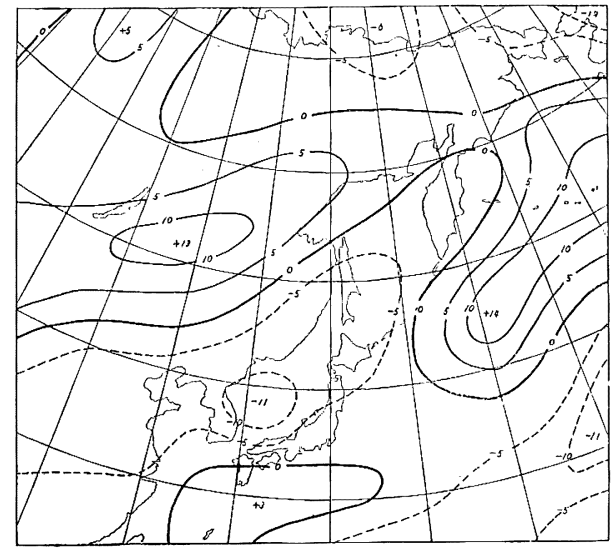

(a) April

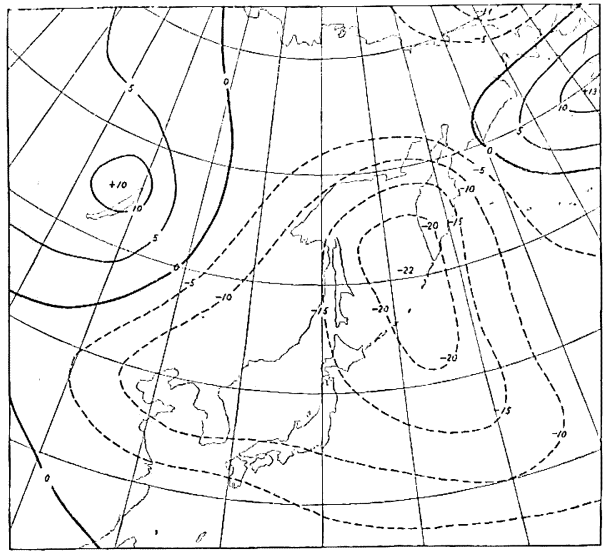

(c) July

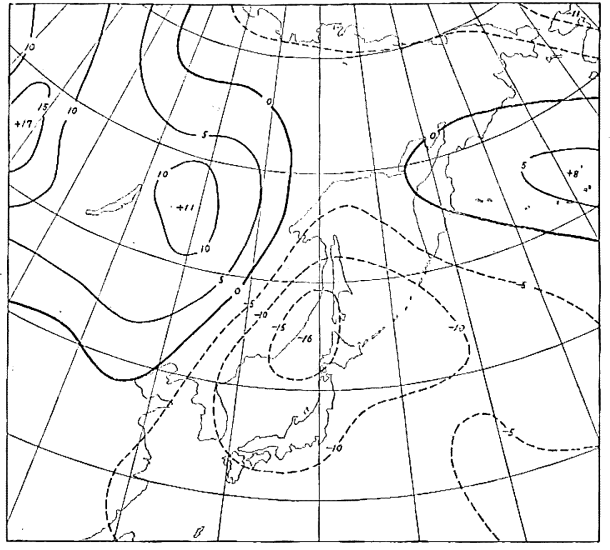

(b) May

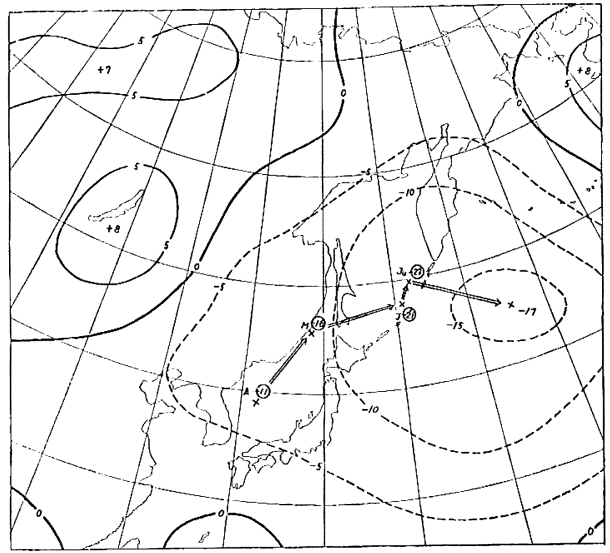

(d) August

Fig. 9. Mean amount of heat at $850 \mathrm{mb}$. Dashed lines are drawn for cooling and continuous lines, for heating. Isolines are for $5 \times 10^{-2} \cdot \mathrm{cal} \cdot \mathrm{gr}^{-1} \cdot 12 \mathrm{hr}^{-1}$ intervals.

It is, here, worthwhile to notice that the magnitude of heating source is rather small in August, i. e. less than $8 \times 10^{-2}$ (cal gr-1 $12 \mathrm{hr}^{-1}$ ).

It is apparent in the five figures $7 \mathrm{~b}, 9 \mathrm{a}, 9 \mathrm{~b}, 9 \mathrm{c}$ and $9 \mathrm{~d}$ that cooling source predominates over the oceans throughout the period from spring to summer. In April, the center of cooling source is found over the central part of the Japan Sea, though the distributions of heating and cooling sources are somewhat complicated over the Pacific. In May, the center of cooling source shifts to the northern Japan Sea, and its magnitude is $-16 \times 10^{-2}$ (cal gr-1 $12 \mathrm{hr}^{-1}$ ). As is easily seen in Fig. 7 (b), the center of cooling source shifts over the southern Sea of Okhotsk, and its magnitude attains $-21 \times 10^{-2}\left(\mathrm{cal} \mathrm{gr}^{-1} 12 \mathrm{hr}^{-1}\right)$ in June. The location, as well as the magnitude, of cooling source remains almost unchanged till July, as is seen in Fig. 9c. In August, the center of cooling source shifts again eastwards to the position $165^{\circ} \mathrm{E}, 45^{\circ} \mathrm{N}$ and its magnitude is reduced to $-17 \times 10^{-2}$ (cal gr-1 $12 \mathrm{hr}^{-1}$ ). 
As described above, the marked maximum of cooling source centering at the sourthern part of the Sea of Okhotsk and remaining there during the two months of June and July, which is presumably favourable for the formation of Baiu, can be traced throughout the period from spring to summer, though its magnitude attains its maximum value in June and July. Referring to the Atlas of Climatic Charts of the Oceans, it may further be possible to conclude that the occurence, as well as the change in location, of such cooling source depends not so much upon the temperature difference between sea and air. Accordingly we have an impression that the cause and locality of Baiu result not merely from cooling at the surface of the Okhotsk Sea, but also from other sources of cooling.

We shall next discuss the fact that the distribution of $(\bar{Q})_{850}$ in January shows better correspondence with common experience concerning the heating from the warmer sea surface, but that in June shows rather bad correspondence. This fact may be explained as follows. That is, the transport of heat due to vertical eddies is predominant in winter, but is extremely small in a warmer season. If the transport of heat due to vertical eddies is small, the patterns of $(\bar{Q})_{850}$ in a warmer season does not probably depend so much upon the cooling from the sea surface.

The theoretical relation between the transports of heat due to vertical and horizontal eddies was derived by A. Arakawa [10] in the course of the discussion about the maintenance of general circulation. His method will be outlined hereafter.

Let each of the quantities-for example the potential temperature-be represented by its mean value with respect to the latitude circle $\bar{\theta}$ plus a departure from this mean $\sigma^{\prime}$, the thermodynamic equation may be written as follows :

$$
\begin{aligned}
\frac{\partial \theta^{\prime}}{\partial t}+ & +U \frac{\partial \theta^{\prime}}{\partial x}+v^{\prime} \frac{\partial \bar{\theta}}{\partial y}+\omega^{\prime} \frac{\partial \bar{\theta}}{\partial p}+\bar{v} \frac{\partial \theta^{\prime}}{\partial y}+\bar{\omega} \frac{\partial \theta^{\prime}}{\partial p} \\
& +\frac{\partial \vec{\theta}}{\partial t}+\bar{v} \frac{\partial \bar{\theta}}{\partial y}+\bar{\omega} \frac{\partial \bar{\theta}}{\partial p}+\left(u^{\prime} \frac{\partial \theta^{\prime}}{\partial x}+v^{\prime} \frac{\partial \theta^{\prime}}{\partial y}+\omega^{\prime} \frac{\partial \theta^{\prime}}{\partial p}\right)=q,
\end{aligned}
$$

in which $U$ is the mean zonal speed, $q$ the heat added to air. Multiplying each term in the above equation by $\theta^{\prime}$ and taking the mean with respect to the latitude circle, the following equation is obtained:

$$
\begin{aligned}
\frac{\partial \overline{\left(\bar{\theta}^{\prime}\right)^{2}}}{\partial t} & +\overline{v^{\prime} \theta^{\prime}} \frac{\partial \bar{\theta}}{\partial y}+\overline{\omega^{\prime} \theta^{\prime}} \frac{\partial \bar{\theta}}{\partial p}+\frac{1}{2}\left[\bar{v} \frac{\partial \overline{\left(\theta^{\prime}\right)^{2}}}{\partial y}+\bar{\omega} \frac{\partial \overline{\left(\theta^{\prime}\right)^{2}}}{\partial p}\right] \\
& +\frac{1}{2}\left[\overline{u^{\prime} \frac{\partial\left(\theta^{\prime}\right)^{2}}{\partial x}}+\overline{v^{\prime} \frac{\partial\left(\theta^{\prime}\right)^{2}}{\partial y}}+\overline{\omega^{\prime} \frac{\partial\left(\theta^{\prime}\right)^{2}}{\partial p}}\right]=0 .
\end{aligned}
$$

Here use is made of the fact that in all cases the mean of any departure shall vanish, and the term $\overline{\theta^{\prime} q}$ is assumed to be zero. In the above equation, $\bar{v}$ and $\bar{\omega}$ are usually quite small, and then the terms containing these quantities may be neglected. The last term in the above equation expresses the triple correlation between $\theta^{\prime}$ and the departures of wind speeds in each direction, and may be assumed to be negligible as far as its statistical value is concerned. Introducing the approximations mentioned above and omitting the first term too, the above equation may now be expressed as

$$
\overline{v^{\prime} \theta^{\prime}} \frac{\partial \bar{\theta}}{\partial y}+\overline{\omega^{\prime} \theta^{\prime}} \frac{\partial \bar{\theta}}{\partial p}=0
$$


This equation may be rewritten as follows:

$$
\overline{\omega^{\prime} \theta^{\prime}}=-\frac{\partial \bar{\theta}}{\partial y} / \frac{\partial \bar{\theta}}{\partial p} \overline{v^{\prime} \theta^{\prime}} \text {. }
$$

It is apparent in the above eqation that the transport of heat due to vertical eddies $\overline{\left(\omega^{\prime} \theta^{\prime}\right)}$ has a linear dependence with that due to horizontal eddies $\left(\overline{\left.v^{\prime} \theta^{\prime}\right)}\right.$, and the proportional constant between these quantities is determined by the slope of the mean isentropic surface. Generally speaking, the slope of the mean isentropic surface is larger in a colder season, and is smaller in a warmer season. So it may be said that the transport of heat due to vertical eddies is predominant in a colder season. In a warmer season, however, the transport of heat due to horizontal eddies is supposed to be significant as compared with that due to vertical eddies.

Referring to the discussion described above, we may be able to suggest that the distributions of $(\bar{Q})_{850}$ in June do not depend very much upon the conditions adjacent to the sea surface. In other words, it is impossible to attribute the cause of Baiu to cooling from the cold sea of Okhotsk alone, though the discussion above is merely proposed as a tentative answer. More complete discussion concerning the cause of Baiu must be left for the future.

Acknowledgements - The writer wishes to express his hearty thanks to Dr. H. Arakawa and Dr. S. Syōno for their guidance throughout the course of this investigation. He is indebted to Mr. A. Arakawa, and the staff members of. N.W.P. group in Tokyo for their discussions kindly exchanged with him. He also wishes to thank Mrs. Y. Tsuneoka for her laborious work of tracing the figures.

\section{References}

[1] Okada, T., 1910: On the Baiu or Rainy Season in Japan, Bull. Cent. Meteor. Obs. Japan, 5, p. 1.

[2] Jacoss, W., 1951: The Energy Exchange between Sea and Atmosphere and Some of its Consequences, Bull. Scripps. Inst. Oceanog. Univ. Cal., 6, p. 7.

[3] Wexuer, M, H., 1944: Determination of the Normal Regions of Heating and Cooling in the Atmosphere by Means of Aerological Data, J. Meteor., 1, p. 23.

[4] Auberm, E, J. and Winston, J, S., 1951: A Study of Atmospheric Heat-Sources in the Northern Hemisphere for Monthly Periods, J. Meteor., 8, p. 111.

[5] Mintz. Y., 1955. An Empirical Determination of Surface Drag Coefficients for Extended-Range and Long-Range Numerical Weather Forecasting.

[6] Cuapr, P, F., 1956: Some Considerations Involved in Preparing Long-Range Forecasts by Numerical Methods, J. Meteor., 13, p. 341.

[ 7 ] Syōnó, S., 1944: Approximate Solution of Non-Linear Differential Equation of Stationary Wind in Axial-Symmetric Cyclone and Anticyclone and Its Applications, Journ. Met. Soc. Japan, 22, p. 366.

[8] Berkofsky, L. and Bertoni, E, A., 1955: Mean Tapographic Charts for the Entire Hemisphere, Bull. Am. Meteor. Society, 36, p. 350.

[ 9 ] Murakami, T., 1953: On the Seasonal Variation of Upper Flow patterns. Part 1. From Winter to Spring, Journ. Met. Soc. Japan. 31, p. 173.

[10] Arakawa, A., 1956: (Unpublished Paper). 